\title{
Novel Fractal DGSs for Band-gap Filter with Improved $Q$-factor
}

\author{
Yan-Yun GONG, Ling WANG, Kun WEI, Zhao-Lin ZHANG
}

School of Electronics and Information, Northwestern Polytechnical University, 710072 Xi'an, Shannxi, China

yanyun_gong@mail.nwpu.edu.cn, lingwang@nwpu.edu.cn,weikun916@163.com, z184@sina.com

Submitted January 11, 2017 / Accepted September 9, 2017

\begin{abstract}
This paper presents a novel fractal defected ground structure (FDGS) for designing high Q-factor band-gap filter. Key parameters of the proposed FDGS are studied. The decreased slot distance causes rapid increase in the filter $Q$-factor. The designed second iterative FDGS provides the band-gap property at $1.662 \mathrm{GHz}$ with 3-dB bandwidth of $10.8 \mathrm{MHz}$ and $Q$-factor 69.25. The rejection level of the second iterative FDGS is greater than $40 \mathrm{~dB}$. The third iterative FDGS has smaller dimension than that of the second iterative FDGS. Moreover, the third iterative FDGS has two resonant frequencies with suppression levels of $33 \mathrm{~dB}$ and $26 \mathrm{~dB}$. The $Q$-factors of the resonant frequencies are 24.22 and 27.36 respectively.
\end{abstract}

\section{Keywords}

High $Q$-factor, band-gap filter, defected ground structure, fractal structure

\section{Introduction}

Many researches on the DGS band-gap filters have been reported with various configurations [1-5]. The DGS is advantageous in the design of microwave and millimeter-wave circuits since it can be modeled by simple resonant circuits and it has simple parameter extraction. The DGS provides rejection band in some frequency range due to providing the effective inductance and effective capacitance of the transmission line [6].

The band-gap filters are developed by using DGS for the suppression of unwanted signals. To suppress the unwanted signal, which is closely located to the desired signal in the spectrum, it is necessary to use the high $Q$-factor band-gap filters. The characteristics of the conventional DGSs with a dumbbell-shaped slot are not satisfactory in the applications of narrowband rejection [7]. The U-slot and V-slot DGSs for band-gap filter with improved $Q$-factor are presented in [6]. The $Q$-factor of the U-slot DGS increases when the distance between two slots in the U-shape decreases. Similarly, the V-slot DGS provides a higher $Q$-factor when the slot angle is reduced. The $Q$-factors of the U-slot and V-shot DGSs are 38.6 and 27.6 respectively. A compact wideband high-rejection filter using H-shaped DGS is presented in [8], the calculated $1.21 Q$-factor is achieved. Another H-shaped compact DGS filter with high $Q$-factor (24.07) is presented in [9]. The slot-shaped DGS on the microstrip line providing a bandgap property with an improved $Q$-factor is proposed in [10]. The DGS unit section provides a $Q$-factor of more than 15, which is 7.6 times than that of the dumbbell DGS. A patch loaded slot-line providing a band-gap property with an improved $Q$-factor (13.9) is proposed in [11]. The $Q$-factor is more than 10.5 times than that of the dumbbell filter at the same resonant frequency. Two C-shaped DGSs are proposed in [12], which have parallel resonant frequencies at $2.468 \mathrm{GHz}$ and $6.629 \mathrm{GHz}$ with high $Q$-factors. The first resonant frequency exhibits $Q$-factor of 65 , while the second resonant frequency $Q$-factor is 63.1. However, the dual-band properties are achieved by two different C-shaped DGSs.

The $Q$-factors and relative bandwidth of the proposed second iterative FDGS in this paper and other high $Q$-factor band-gap DGS filters are summarized and compared in Tab. 1. Comparing to other band-gap filters in the reference list, the designed second iterative FDGS band-gap filter has the narrowest relative bandwidth and the highest measured $Q$-factor.

When designing a planar band-gap DGS filter, a minimal circuit dimension is desired. Fractal structures enable us to create longer current lines on a smaller surface, which

\begin{tabular}{|c|c|c|c|c|}
\hline & $\begin{array}{c}\text { Resonant } \\
\text { frequency } \\
(\mathrm{GHz})\end{array}$ & $\begin{array}{c}\text { 3-dB } \\
\text { bandwidth } \\
(\mathrm{GHz})\end{array}$ & $\begin{array}{c}\text { Relative } \\
\text { bandwidth }\end{array}$ & Q-factor \\
\hline Ref. [6] & 2.475 & 0.064 & $2.59 \%$ & 38.6 \\
\hline Ref. [7] & 3.45 & 2.95 & $85.5 \%$ & 1.17 \\
\hline Ref. [8] & 14 & 11.6 & $82.85 \%$ & 1.21 \\
\hline Ref. [9] & 1.685 & 0.07 & $4.15 \%$ & 24.07 \\
\hline Ref. [10] & 5.3 & 0.34 & $6.42 \%$ & 15.59 \\
\hline Ref. [11] & 32.9 & 2.37 & $7.2 \%$ & 13.9 \\
\hline Ref. [12] & 2.469 & 0.038 & $1.54 \%$ & 65 \\
\hline This work & 1.662 & 0.024 & $1.44 \%$ & 69.25 \\
\hline
\end{tabular}

Tab. 1. Calculated $Q$-factor comparison of DGSs. 
reduces the structure dimensions. The filters using the Koch-shaped fractal are proposed in [13], which can be used to suppress the second harmonic without any additional devices. The Minkowski fractal structure is inserted in different sections of a dumbbell-shaped DGS, higher iteration of the fractals gives good results on the cost of increasing the structure complexity [14]. The Koch fractal on the dumbbell-shaped DGS filter is used to improve the filter performance, increase the frequency selectivity, and reduce the filter size [15]. The Hilbert fractal DGS unit [16], [17] has a smaller dimension, which reduces the passband reflection loss and restrains higher order harmonic components. The fractal DGS filter has a $20 \%$ smaller substrate compared to a conventional filter with stubs [18]. All these fractal structures aim to reduce the DGS dimension.

In this paper, the authors propose a novel FDGS band-gap filter with high $Q$-factor. The key parameters of the proposed fractal structure are studied. The decreased slot distance causes rapid increase in the $Q$-factor. The $Q$-factor of the second iterative FDGS band-gap filter is higher than that of the conventional DGSs. High level iterative fractal structure creates longer current lines on a smaller surface to reduce the structure dimension. Therefore, the third iterative FDGS has lower resonant frequency compared to that of the second iterative FDGS when they have the same outline dimension.

This paper is organized as follows. The structure of the proposed FDGS is given in Sec. 2. In Sec. 3, the parameter studies of the second iterative FDGS and its measured results are presented. In Sec. 4, the performance comparison between the second and third iterative FDGS, the measured results of the third iterative FDGS are presented. A conclusion is given in Sec. 5 .

\section{FDGS Geometry}

The geometries of the proposed fractal of zero, first, second and third iterative fractal structures are shown in Fig. 1. The zero iterative structure is a single line as shown in Fig. 1(a). Two basic U-shaped structures are added to two endpoints of the zero iterative. In this way, the zero iterative structure is turned into the first iterative structure as shown in Fig. 1 (b). Now, the first iterative structure has four endpoints. Adding four basic U-shaped structures to the four endpoints of the first iterative structure transforms it to the second iterative structure, as shown in Fig. 1(c). The third iterative fractal structure, shown in Fig. 1(d), evolves from the second iterative fractal structure by adding eight basic U-shaped structures to the eight endpoints of the second iterative structure. By repeating this process, high level iterative fractal structure is designed.

The designed fractal structure is more flexible compared to the Minkowski or Koch fractal structures. We add

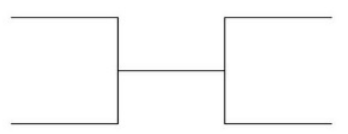

(a)

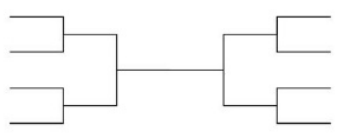

(c) (b)

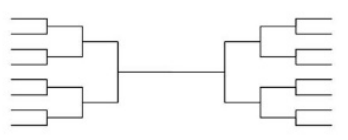

(d)
Fig. 1. Designed fractal geometries; (a) zero iteration (b) first iteration (c) second iteration (d) third iteration.

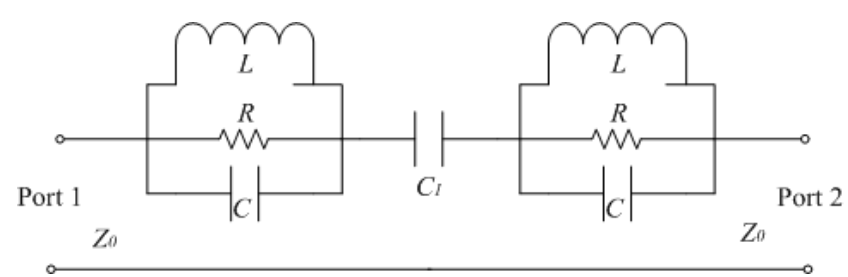

Fig. 2. Equivalent circuit of the DGS.

U-shaped unit into the structure for achieving higher iterative fractal structure. The designed fractal structure has self-similar pattern. Unlike the Minkowski or Koch fractals, the U-shaped units for different iterative fractal structures do not have to be proportional to each other. Hence, the designed fractal DGS has more parameters to adjust for optimizing the band-gap filter performances.

The equivalent model of the designed FDGS has been extracted to present insight to this kind of DGS as shown in Fig. 2. The frequency characteristic of the proposed FDGS can be modeled by two parallel resonant circuits in the transmission line to block the signal transfer at the resonant frequency. The resonant circuit is formed by a parallel RLC in the transmission line. Since the fractal structure is symmetrical, the two parallel RLC resonant circuits are the same. The DGSs are connected by a rectangular slot, so the two parallel RLC are in series with capacitance $C_{1}$.

One of the $Q$ definitions is the frequency-to-bandwidth ratio of the resonator. Thus, in the parallel resonant circuit, the $Q$-factor is proportional to the parameter $\sqrt{C / L}$.

The circuit parameters of the equivalent circuit from the simulated scattering parameters as given in [19]:

$$
\begin{gathered}
C=\frac{\omega_{\mathrm{c}}}{2 Z_{0}\left(\omega_{0}^{2}-\omega_{\mathrm{c}}^{2}\right)}, \\
L=\frac{1}{4 \pi^{2} f_{0}^{2} C}
\end{gathered}
$$

where $\omega_{0}$ is the angular resonant frequency, $\omega_{\mathrm{c}}$ is the $3-\mathrm{dB}$ cutoff angular frequency, $f_{0}$ is the resonant frequency, and $Z_{0}$ is the characteristic impedance of the microstrip line. 


\section{The Second Iterative FDGS Characteristics}

The configuration of the proposed second iterative FDGS in the ground plane is shown in Fig. 3. The gap width of the FDGS is $g$. The distances between slots are $d 1$ and $d 2$ respectively. The substrate has dielectric constant 2.65 and thickness $1 \mathrm{~mm}$. For the dimensions of $l 1=3.5 \mathrm{~mm}, \quad l 2=12 \mathrm{~mm}, \quad l 3=15 \mathrm{~mm}, \quad g=0.1 \mathrm{~mm}$, $d 1=0.4 \mathrm{~mm}$ and $d 2=0.1 \mathrm{~mm}, \mathrm{~S} 11$ and $\mathrm{S} 21$ of the second iterative FDGS are optimized. The impedance of the microstrip line above the FDGS is assumed to be $50 \Omega$ and the simulation is performed by using Ansoft High Frequency Structure Simulator (HFSS). In Fig. 4, the S11 and S21 are plotted as a function of frequency. The designed second iterative FDGS provides the band-gap property at $1.662 \mathrm{GHz}$ with $3-\mathrm{dB}$ bandwidth of $24 \mathrm{MHz}$, and cutoff frequency is $1.650 \mathrm{GHz}$, where the calculated circuit parameters are $C=66.11 \mathrm{pF}, L=0.14 \mathrm{nH}$. Thus, the calculated $Q$-factor of the proposed second iterative FDGS is 69.25. Moreover, the investigated second iterative FDGS has a rejection level better than $40 \mathrm{~dB}$.

The characteristic of the second iterative FDGS such as rejection bandwidth, resonant frequency and $Q$-factor are dependent on the dimensions of the FDGS. The gap length, gap width, slot distance are the key parameters of the proposed second iterative FDGS. In order to study the FDGS dimensions, simulated transfer characteristics for the second iterative FDGS as functions of key parameters are plotted from Fig. 5 to Fig. 7. In Fig. 5, the simulated transfer characteristics are plotted as a function of gap length ( $l 3)$. The values of the rest parameters are $l 1=$ $2.5 \mathrm{~mm}, l 2=12 \mathrm{~mm}, g=0.15 \mathrm{~mm}, d 1=1.85 \mathrm{~mm}$ and $d 2=$ $0.9 \mathrm{~mm}$. It is clearly seen that the increased gap length $l 3$ decreases the resonant frequency. This is because that the increased gap length increases the effective inductance and increases the effective capacitance, which gives rise to a lower cutoff frequency. The $Q$-factors with different gap length are also calculated and shown in Tab. 2.

It can be seen that the change in the calculated $Q$-factor has no relevance with gap length 13 . This is because that the $Q$-factor is proportional to parameters $C$ and $1 / L$, adding that the increased gap length increases the effective

\begin{tabular}{|l|c|c|c|c|}
\hline Parameter (mm) & $l 3=8$ & $l 3=11$ & $l 3=15$ & $l 3=18$ \\
\hline Resonant frequency (GHz) & 2.053 & 1.777 & 1.561 & 1.399 \\
\hline Cutoff frequency (GHz) & 1.871 & 1.611 & 1.413 & 1.269 \\
\hline 3-dB bandwidth (GHz) & 0.364 & 0.332 & 0.296 & 0.26 \\
\hline Calculated $Q$-factor & 5.64 & 5.35 & 5.28 & 5.38 \\
\hline Capacitance (pF) & 4.17 & 4.56 & 5.11 & 5.82 \\
\hline Inductance (nH) & 1.44 & 1.76 & 2.03 & 2.22 \\
\hline Suppression level (dB) & 32 & 45 & 40 & 35 \\
\hline
\end{tabular}

Tab. 2. The DGSs performances when tuning gap length $l 3$.

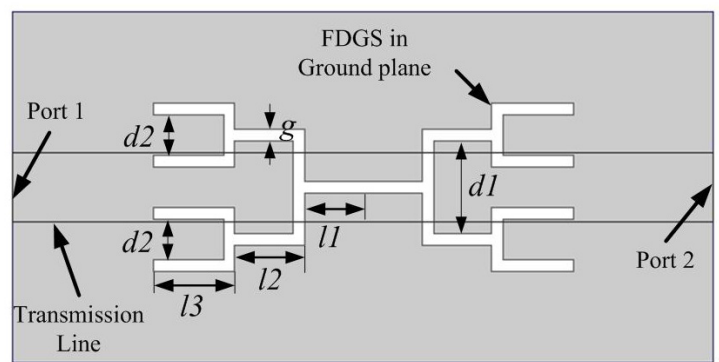

Fig. 3. The proposed novel second iterative FDGS band-gap filter.

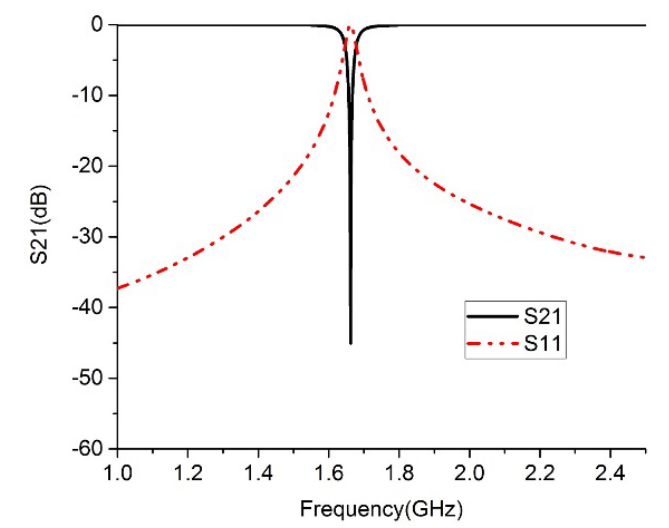

Fig. 4. S parameters of the optimized second iterative FDGS $(l 1=3.5 \mathrm{~mm}, l 2=12 \mathrm{~mm}, l 3=15 \mathrm{~mm}, g=0.1 \mathrm{~mm}$, $d 1=0.4 \mathrm{~mm}, d 2=0.1 \mathrm{~mm})$.

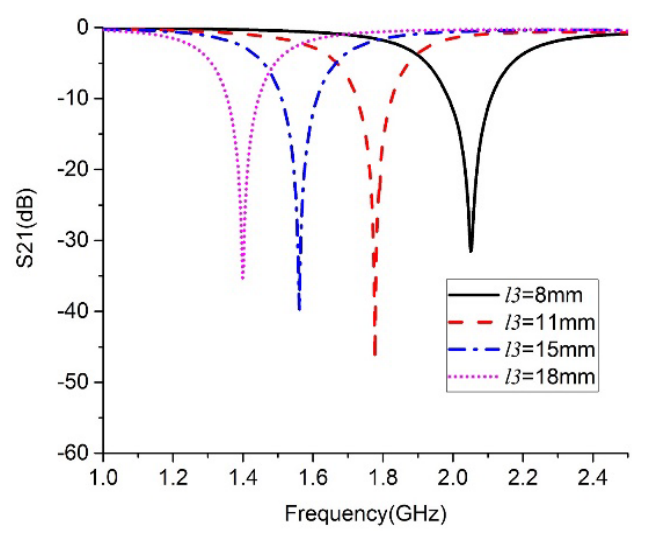

Fig. 5. Transfer characteristics for various gap lengths $(l 3)$ of the proposed second iterative FDGS $(l 1=2.5 \mathrm{~mm}, l 2=$ $12 \mathrm{~mm}, g=0.15 \mathrm{~mm}, d 1=1.85 \mathrm{~mm}, d 2=0.9 \mathrm{~mm})$.

inductance and increases the effective capacitance. The suppression levels of the resonant frequencies with different gap length are smaller than $30 \mathrm{~dB}$. The same conclusion can be drawn when tuning other gap lengths $l 1$ and $l 2$.

The simulated S21 of the proposed second iterative FDGS with different gap widths are shown in Fig. 6. The gap width changes from $0.15 \mathrm{~mm}$ to $1 \mathrm{~mm}$, the values of the rest parameters are $l 1=3.5 \mathrm{~mm}, l 2=12 \mathrm{~mm}, l 3=$ $15 \mathrm{~mm}, d 1=4.7 \mathrm{~mm}$ and $d 2=2.3 \mathrm{~mm}$. As the gap width increases, the effective capacitance decreases and the effective inductance increases. The $Q$-factors with the different gap widths are calculated and shown in Tab. 3. The increased gap width decreases the effective capacitance and 


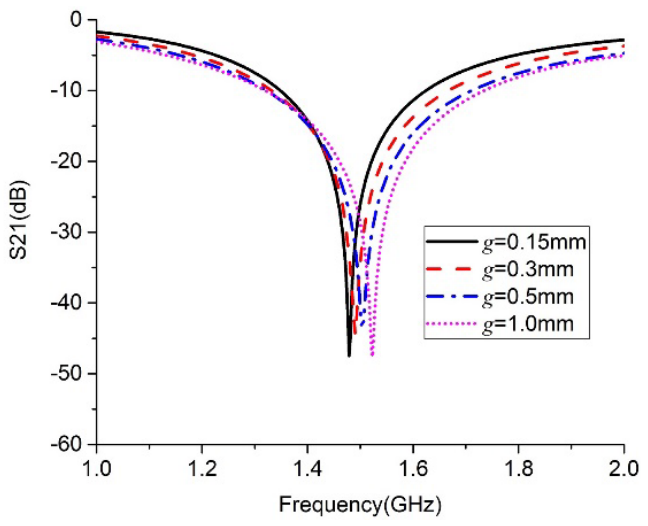

Fig. 6. Transfer characteristics for various gap widths $(g)$ of the proposed second iterative FDGS $(l 1=3.5 \mathrm{~mm}$, $l 2=12 \mathrm{~mm}, l 3=15 \mathrm{~mm}, d 1=4.7 \mathrm{~mm}, d 2=2.3 \mathrm{~mm})$.

\begin{tabular}{|l|c|c|c|c|}
\hline Parameter (mm) & $g=0.15$ & $g=0.3$ & $g=0.5$ & $g=1$ \\
\hline Resonant frequency (GHz) & 1.479 & 1.491 & 1.505 & 1.523 \\
\hline Cutoff frequency (GHz) & 1.123 & 1.067 & 1.025 & 0.993 \\
\hline 3-dB bandwidth (GHz) & 0.712 & 0.848 & 0.96 & 1.06 \\
\hline Calculated $Q$-factor & 2.08 & 1.76 & 1.57 & 1.44 \\
\hline Capacitance (pF) & 1.93 & 1.67 & 1.34 & 1.19 \\
\hline Inductance (nH) & 6 & 6.82 & 8.35 & 9.18 \\
\hline Suppression level (dB) & 46 & 45 & 44 & 46 \\
\hline
\end{tabular}

Tab. 3. The DGSs performances when tuning gap width $g$.

increases the effective inductance, adding that the $Q$-factor is proportional to the parameter $\sqrt{C / L}$, so that the increased gap width will decrease the calculated $Q$-factor. The suppression levels of the resonant frequencies with different gap widths are lower than $40 \mathrm{~dB}$.

The simulated S21 of the proposed second iterative FDGS as a function of frequency with different slot distances $d 1$ and $d 2$ are plotted in Fig. 7, when the rest parameter values are $l 1=3.5 \mathrm{~mm}, l 2=12 \mathrm{~mm}, l 3=15 \mathrm{~mm}$ and $g=0.15 \mathrm{~mm}$. The decreased slot distances increase the effective capacitance and decrease the effective inductance as shown in Tab. 4. The calculated $Q$-factors with decreased slot distances $d 1$ and $d 2$ lead to increase in the $Q$ factor, which has been proven by the simulations as given in Tab. 4. Moreover, the decreased slot distance causes rapid increase in the $Q$-factor compared to that of the structure gap length and gap width. The suppression levels of the resonant frequencies greater than $35 \mathrm{~dB}$ with different slot distances are achieved.

Figure 8 shows the top view and bottom view of the fabricated second iterative FDGS. The dielectric constant of chosen substrate is 2.65 , and the thickness is $1 \mathrm{~mm}$. The ports of the transmission line are connected with two SMA connectors. The proposed second iterative FDGS is etched away from the ground plane. Comparisons of the S11 and S21 between simulation and measurement are indicated in Fig. 9. Some deviations of the measurement from the simu-

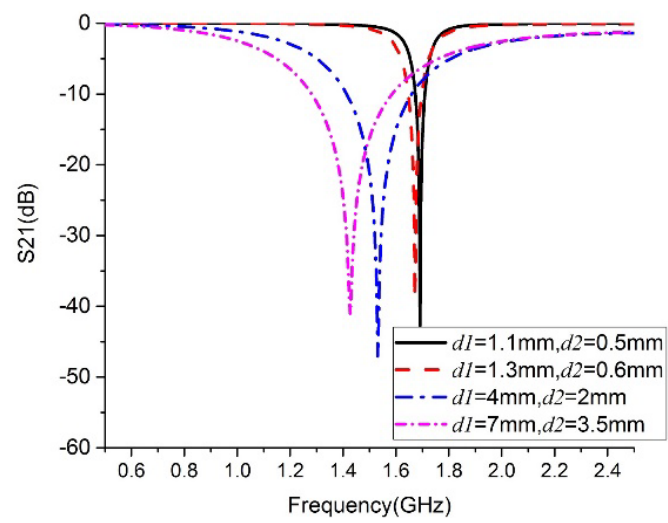

Fig. 7. Transfer characteristics for various U-slot distances $(d 1, d 2)$ of the proposed second iterative FDGS $(l 1=3.5 \mathrm{~mm}, l 2=12 \mathrm{~mm}, l 3=15 \mathrm{~mm}, g=0.15 \mathrm{~mm})$.

\begin{tabular}{|l|c|c|c|c|}
\hline Parameter $(\mathrm{mm})$ & $\begin{array}{c}d 1=7, \\
d 2=3.5\end{array}$ & $\begin{array}{c}d 1=4, \\
d 2=2\end{array}$ & $\begin{array}{c}d 1=1.3, \\
d 2=0.6\end{array}$ & $\begin{array}{c}d 1=1.1, \\
d 2=0.5\end{array}$ \\
\hline Resonant frequency $(\mathrm{GHz})$ & 1.427 & 1.531 & 1.671 & 1.691 \\
\hline Cutoff frequency $(\mathrm{GHz})$ & 1.014 & 1.205 & 1.595 & 1.635 \\
\hline 3-dB bandwidth $(\mathrm{GHz})$ & 0.826 & 0.652 & 0.152 & 0.112 \\
\hline Calculated $Q$-factor & 1.73 & 2.35 & 10.99 & 15.1 \\
\hline Capacitance (pF) & 1.6 & 2.15 & 10.23 & 13.98 \\
\hline Inductance $(\mathrm{nH})$ & 7.77 & 5.03 & 0.89 & 0.63 \\
\hline Suppression level $(\mathrm{dB})$ & 40 & 46 & 37 & 43 \\
\hline
\end{tabular}

Tab. 4. The DGSs performances when tuning slot distance.

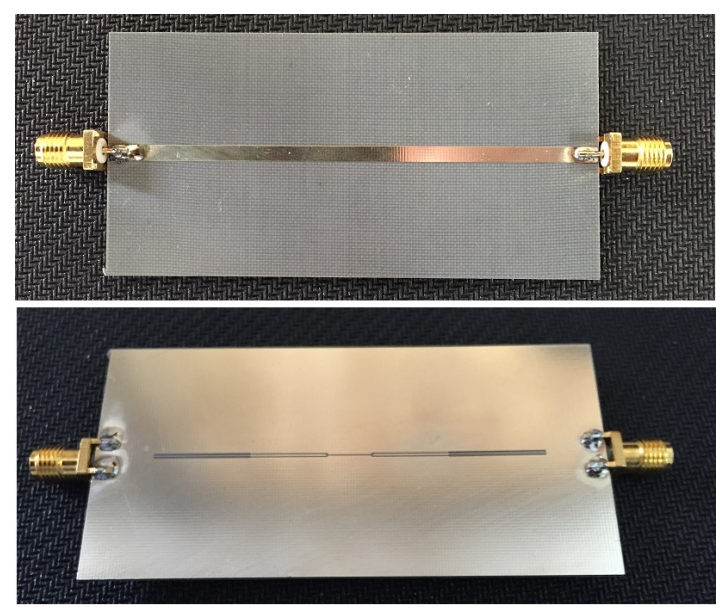

Fig. 8. Bottom view and top view of the proposed second iterative FDGS.

lation occur in the stop-band, which are mainly due to the reflections from the connectors. The measured resonant frequency is $1.698 \mathrm{GHz}$, and the measured 3-dB bandwidth of the second iterative FDGS is about $26 \mathrm{MHz}$. The calculated $Q$-factor of the fabricated second iterative FDGS is 65.31. The measurement shows that the fabricated second iterative FDGS filter with the proposed structure has steep band-gap property and the filter provides high $Q$-factor. Note that the measured S21 is shifted to higher frequency region. Except for the allowable measurement errors, the 


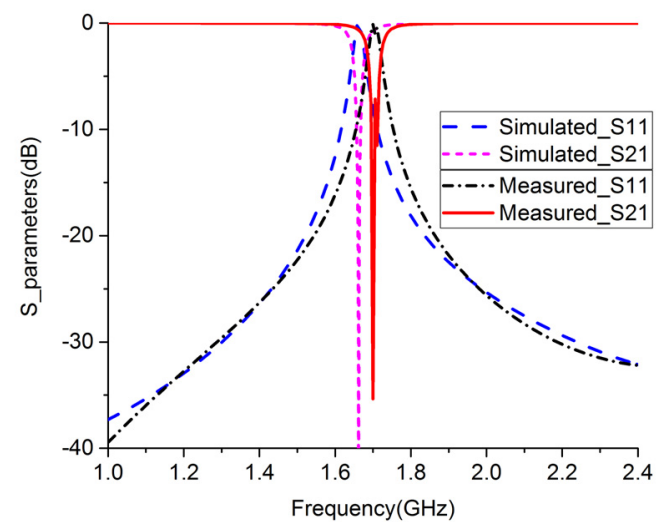

Fig. 9. The simulation and measurement results of the proposed second iterative FDGS.

reason for the difference between simulation and measurement is mainly caused by the machine error of the fabricated second iterative FDGS.

\section{Third Iterative FDGS Characteristics}

The geometries of the proposed third iterative FDGS is shown in Fig. 10. The gap width of the third iterative FDGS is $g$. The slot distances are $d 1, d 2$ and $d 3$ differently. The dielectric constant of substrate is 2.65 with thickness $1 \mathrm{~mm}$. S11 and S21 of the third iterative FDGS are optimized for the values of $l 1=2.5 \mathrm{~mm}, l 2=5 \mathrm{~mm}, l 3=$ $11 \mathrm{~mm}, \quad l 4=11 \mathrm{~mm}, g=0.15 \mathrm{~mm}, d 1=0.65 \mathrm{~mm}, d 2=$ $0.25 \mathrm{~mm}$ and $d 3=0.05 \mathrm{~mm}$.

In Fig. 11, S11 and S21 of the third iterative FDGS are plotted as a function of frequency. The second resonant frequency occurs when the second iterative FDGS is transformed to the third iterative FDGS. This may be caused by that the third iterative FDGS adds another new parallel RLC resonant circuit to the equivalent circuit of the second iterative FDGS. The third iterative FDGS has lower resonant frequency $1.405 \mathrm{GHz}, 3-\mathrm{dB}$ bandwidth about $58 \mathrm{MHz}$, and cutoff frequency $1.376 \mathrm{GHz}$, which means the circuit parameters are $C=27.17 \mathrm{pF}$ and $L=0.47 \mathrm{nH}$ respectively. The calculated $Q$-factor of the lower resonant frequency is about 24.22. The higher resonant frequency of the third iterative FDGS is $1.97 \mathrm{GHz}$ with $3-\mathrm{dB}$ bandwidth

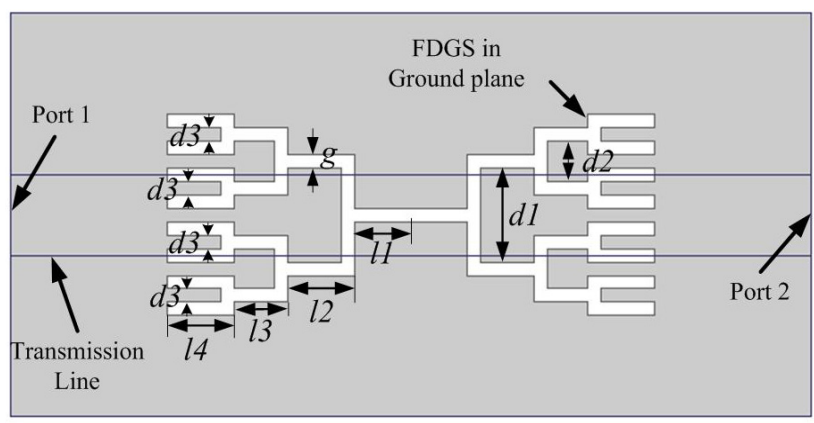

Fig. 10. The geometries of the proposed novel third iterative FDGS band-gap filter.

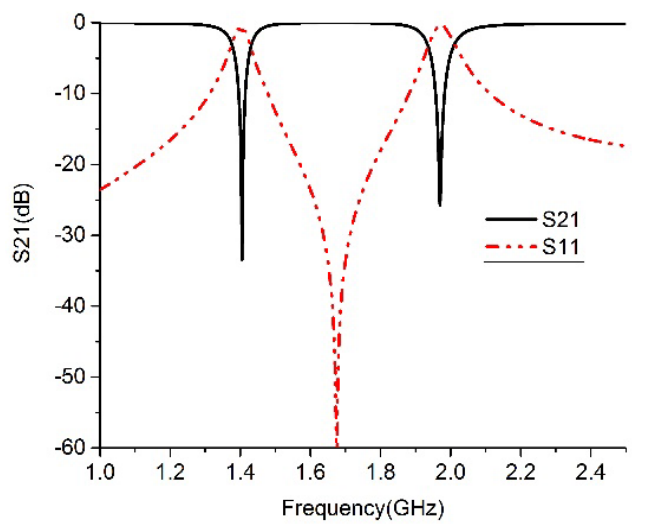

Fig. 11. S parameters of the optimized third iterative FDGS $(l 1=2.5 \mathrm{~mm}, l 2=5 \mathrm{~mm}, l 3=11 \mathrm{~mm}, l 4=11 \mathrm{~mm}$, $g=0.15 \mathrm{~mm}, d 1=0.65 \mathrm{~mm}, d 2=0.25 \mathrm{~mm}, d 3=$ $0.05 \mathrm{~mm}$.)

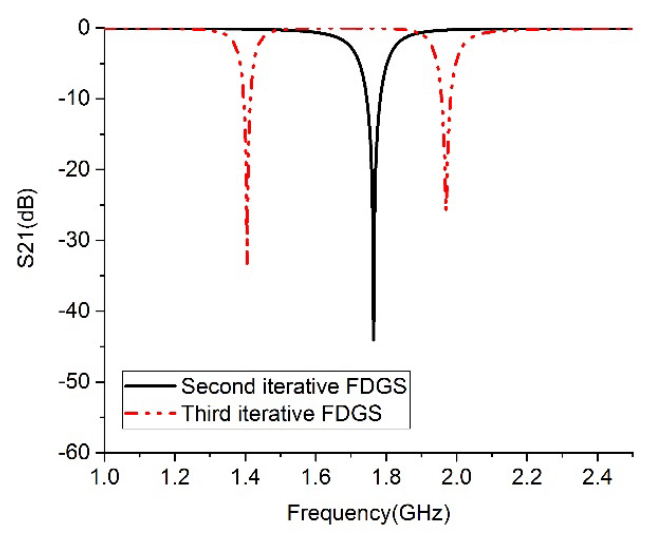

Fig. 12. S21 comparison between the second and the third iterative FDGS.
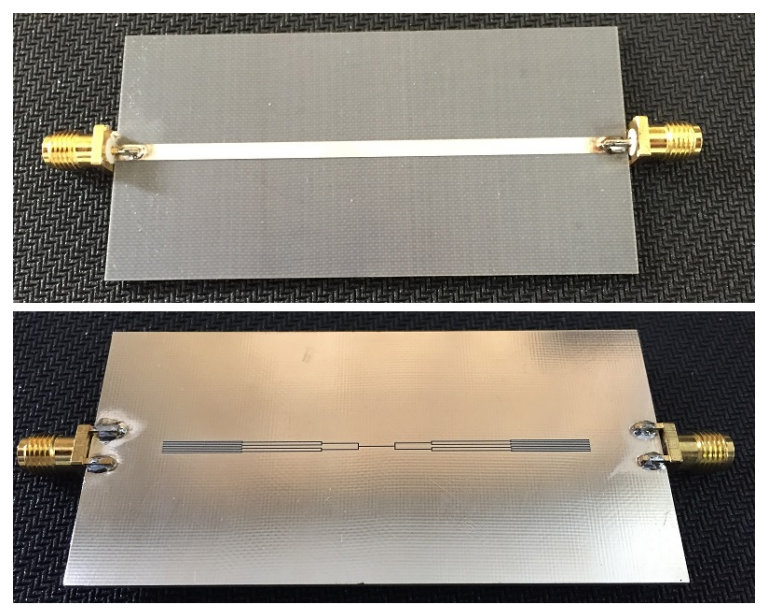

Fig. 13. Bottom view and top view of the proposed third iterative FDGS.

approximately $72 \mathrm{MHz}$, where the circuit parameters are $C=21.91 \mathrm{pF}$ and $L=0.30 \mathrm{nH}$. The calculated $Q$-factor of the higher resonant frequency is 27.36. The resonant frequencies of the third iteration fractal DGS cannot be controlled independently because the fractal DGS dimension change will affect both resonant frequencies. But we can design the structure with high $Q$-factor and both resonant frequencies close to each other. When we enlarge the struc- 
ture length, the resonant frequencies of the third iterative fractal DGS move to lower region and closer to each other. Moreover, the third iterative fractal DGS has high $Q$-factor when the structure length is changed.

The S21 comparison between the second and the third iterative FDGS is illustrated in Fig. 12. The designed third iterative FDGS and the second iterative FDGS have the same outline dimension. Higher level iterative fractal structure creates longer current line on a smaller surface to reduce the DGS dimensions. Because of that, the first resonant frequency of the third iterative FDGS is lower than that of the second iterative FDGS resonant frequency when they have the same outline dimension. The first resonant frequency of the third iterative FDGS is about $1.415 \mathrm{GHz}$, which shifts $247 \mathrm{MHz}$ to lower frequency region compared to that of the second iterative FDGS. That is to say, about $14.9 \%$ size reduction is achieved by transforming the second iterative FDGS to the third iterative FDGS.

The third iterative FDGS has the same key parameters as the second iterative FDGS, namely gap length, gap width and slot distance. The increased gap length of the third iterative FDGS increases the effective inductance and increases the effective capacitance, which gives rise to the lower cutoff frequency. As the gap width increases, the effective capacitance decreases and the effective inductance increases, this decreases the calculated $Q$-factor. Furthermore, the decreased slot distance $d 1, d 2$ and $d 3$ of the third iterative FDGS will increase the effective capacitance and decrease the effective inductance sharply, which will cause rapid increase in the $Q$-factor.

The top view and bottom view of the fabricated third iterative FDGS are shown in Fig. 13. The substrate has dielectric constant 2.65 and thickness $1 \mathrm{~mm}$, which are the same as the substrate used by the fabricated second iterative FDGS. The third iterative FDGS is etched away from the ground plane. The input and output ports of the $50 \Omega$ impedance transmission line are connected with SMA connectors.

The simulated and measured S-parameters of the proposed third iterative FDGS are illustrated in Fig. 14. The reason for the difference between the simulation and meas-

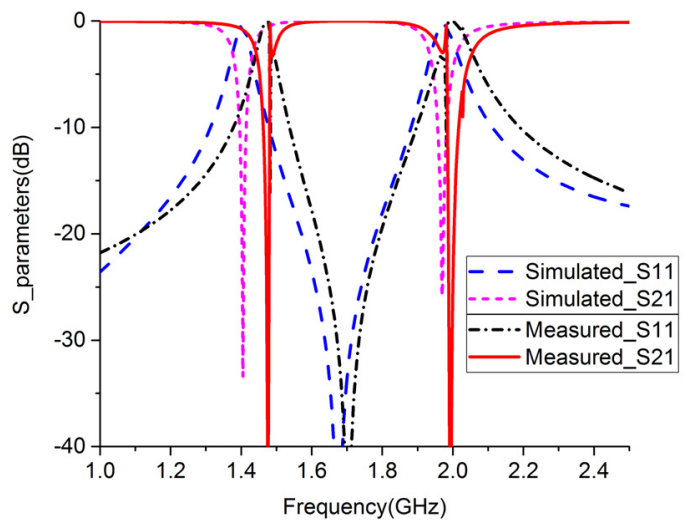

Fig. 14. Simulation and measurement $S$ parameters of the proposed third iterative FDGS. urement, except the allowable measurement errors, is mainly caused by the machine error of the fabricated FDGS, since the FDGS performance is sensitive to its dimensions. The measured third iterative FDGS first resonant frequency is $1.476 \mathrm{GHz}$ with the $3-\mathrm{dB}$ bandwidth $62 \mathrm{MHz}$. The measured $Q$-factor of the third iterative FDGS first resonant frequency is about 23.81. The measured second resonant frequency is about $1.994 \mathrm{GHz}$, and the $3-\mathrm{dB}$ bandwidth is $80 \mathrm{MHz}$. The measured $Q$-factor of the second resonant frequency is about 24.93. The measurement show that the fabricated third iterative FDGS filter has two resonant frequencies with steep band-gap property and it provides high $Q$-factors at two resonant frequencies.

\section{Conclusion}

The novel FDGS band-gap filter with high $Q$-factor is proposed in this paper. The key parameters of the proposed second and the third iterative FDGS are studied. The decreased slot distance causes rapid increase in the $Q$-factor. The simulated and measured $Q$-factors of the second iterative FDGS are 69.25 and 65.31. Comparing to the second iterative FDGS, the third iterative FDGS has smaller dimension. Moreover, the third iterative FDGS has two resonant frequencies. The simulated first resonant frequency exhibits $Q$-factor of 24.22 while the second resonant frequency $Q$-factor is about 27.36 . The measured third iterative FDGS $Q$-factors are 23.81 and 24.93 respectively.

\section{Acknowledgments}

This study was supported in part by the National Natural Science Foundation of China (No.61301093, No. 61601372 and No. 61601373)

\section{References}

[1] DAL AHN, JUN-SEOK PARK, CHUL-SOO KIM, et al. A design of the low-Pass filter using the novel microstrip defected ground structure. IEEE Transactions on Microwave Theory and Techniques, 2001, vol. 49, no. 1, p. 86-93. DOI: $10.1109 / 22.899965$

[2] YU LIN ZHANG, WEI HONG, KE WU, et al. Novel substrate integrated waveguide cavity filter with defected ground structure. IEEE Transactions on Microwave Theory and Techniques, 2005, vol. 53 , no. 4 , p. 1280-1287. DOI: 10.1109/TMTT.2005.845750

[3] JONG-SIK LIM, CHUL-SOO KIM, DAL AHN, et al. Design of low-pass filters using defected ground structure. IEEE Transactions on Microwave Theory and Techniques, 2005, vol. 53, no. 8, p. 2539-2545. DOI: 10.1109/TMTT.2005.852765

[4] SAFWAT, A. M. E., PODEVIN, F., FERRARI, P., et al. Tunable bandstop defected ground structure resonator using reconfigurable dumbbell-shaped coplanar waveguide. IEEE Transactions on Microwave Theory and Techniques, 2006, vol. 54, no. 9, p. 3559 to 3564. DOI: $10.1109 /$ TMTT.2006.880654 
[5] FU-CHANG CHEN, HAO-TAO HU, JIE-MING QIU, et al. Highselectivity low-pass filters with ultrawide stopband based on defected ground structures. IEEE Transactions on Components, Packaging and Manufacturing Technology, 2015, vol. 5, no. 9, p. 1313-1319. DOI: 10.1109/TCPMT.2015.2461663

[6] DUK-JAE WOO, TAEK-KYUNG LEE, JAE-WOOK LEE, et al. Novel U-slot and V-slot DGSs for bandstop filter with improved Q factor IEEE Transactions on Microwave Theory and Techniques, 2006, vol. 54, no. 6, p. 2840-2847. DOI: 10.1109/TMTT.2006.875450

[7] KUMAR, P., MAHMOOD, R., et al. Control of band stop responses of very compact size microstrip filter of improved $Q$ factor and sharp transition by using hexagonal transmetal DGS. In International Conference on Emerging Trends in Electronic and Photonic Devices and Systems (ELECTRO). Varanasi (India), 2009, p. 383-386.

[8] BOUTEJDAR, A., AMARI, S., ELSHERBINI, A., et al. A novel lowpass filter with ultra-wide stopband and improved $Q$-factor performance using $\mathrm{H}$-defected ground structure (DGS). In IEEE Antennas and Propagation Society International Symposium. Honolulu (HI, USA), 2007, p. 1545-1548. DOI: 10.1109/APS.2007.4395802

[9] WANG, C.-J., LIN, C.-S. Compact DGS resonator with improvement of $Q$-factor. Electronics Letters, 2008, vol. 44, no. 15, p. 908-909. DOI: 10.1049/el:20081417

[10] MAOHUI YANG, JUN XU, YULIANG DONG, et al. A novel open-loop DGS for compact bandstop filter with improved $Q$ factor. In 8th International Symposium on Antennas, Propagation and EM Theory. Kunming (China), 2008, p. 649-652. DOI: 10.1109/ISAPE.2008.4735297

[11] MAOHUI YANG, JUN-XU, YULIANG DONG, et al. Bandstop filter with improved $Q$ factor using novel open-loop patch loaded slotline. In 8th International Symposium on Antennas, Propagation and EM Theory. Kunming (China), 2008, p. 1446-1449. DOI: 10.1109/ISAPE.2008.4735502

[12] KAKHKI, M. A., NESHATI, M. H. Experimental investigation of a dual band-reject filter using C-shaped DGS with improved $Q$ factor. In 5th International Symposium on Telecommunications (IST). Tehran (Iran), 2010, p. 332-335. DOI: 10.1109/ISTEL.2010.5734047

[13] IL KWON KIM, KINGSLEY, N., MORTON, M., et al. Fractalshaped microstrip coupled-line bandpass filters for suppression of second harmonic. IEEE Transactions on Microwave Theory and Techniques, 2005, vol. 53, no. 9, p. 2943-2948. DOI: 10.1109/TMTT.2005.854263

[14] KUMAR, A., SAWANT, A., KARTIKEYAN, M. V. Investigation of fractal DGS microwave filters. In National Conference on Communications (NCC). New Delhi (India), 2013, 4 p. DOI: 10.1109/NCC.2013.6487905

[15] CHEN JIA XI, GUAN YA LIN. Application of Koch island fractal on dumbbell-shaped defected ground structure low-pass filters. In IEEE Workshop on Advanced Research and Technology in Industry Applications (WARTIA). Ottawa (Canada), 2014, p. 1101-1104. DOI: 10.1109/WARTIA.2014.6976469
[16] ZHANG WEI, YANG WEI-MING, FANG YIN-YIN, et al. Design of the Hilbert fractal defected ground structure low-pass filter. In IEEE International Conference on Communication Problem-Solving (ICCP). Bejing (China), 2014, p. 99-102. DOI: 10.1109/ICCPS.2014.7062227

[17] HAIWEN LIU, ZHICHONG ZHANG, XUEHUI GUAN, et al. A bandpass filter based on fractal shaped defected ground structure resonators for wireless communication. In International Conference on Microwave and Millimeter Wave Technology. 2010, p. 201-203. DOI: 10.1109/ICMMT.2010.5525249

[18] KUFA, M., RAIDA, Z. Lowpass filter with reduced fractal defected ground structure. Electronics Letters, 2013, vol. 49, no. 3 , p. 199-201. DOI: 10.1049/el.2012.3473

[19] QUAN XUE, KAM MAN SHUM, CHI HOU CHAN. Novel 1-D microstrip PBG cell. IEEE Microwave and Guided Wave Letters, 2000, vol. 10 , no. 10 , p. 403-405. DOI: $10.1109 / 75.877226$

\section{About the Authors ...}

Yan-Yun GONG received the degrees of B.Sc and M.Sc in Xidian University in 2008 and 2012, respectively. Currently he is working on Ph.D degree in the Northwestern Polytechnical University. His recent research interests include microstrip antennas, wireless communication, signal processing, radar system.

Ling WANG received the degrees of B.Sc. M.Sc. and Ph.D. from Xidian University, Xi'an, China, in 1999, 2002 and 2004, respectively, all in Electronic Engineering. From 2004 to 2007, he worked at Siemens and Nokia Siemens Networks. Since 2007, he has been with the School of Electronic and Information, Northwestern Polytechnical University and was promoted to Professor in 2012. His current research interests include array processing and smart antennas, wideband communications, cognitive radio, adaptive anti-jamming for satellite communications, satellite navigation, and date link systems.

Kun WEI received the degrees of B.Sc and M.Sc in Electronic Engineering in the Northwestern Polytechnical University in 2011 and 2014, respectively. Currently he is working on Ph.D degree in the Northwestern Polytechnical University. His recent research interests include microstrip antennas, satellite communication antenna, circularly-polarized antenna, periodic structure, and defected ground structure (DGS).

Zhao-Lin ZHANG is the assistant professor in the Northwestern Polytechnical University. His current research interests include wideband communications, cognitive radio, adaptive anti-jamming for satellite communications and satellite navigation. 